PROSTATE CANCER

\title{
Targeting the ribosome in advanced disease
}

Overexpression of the MYC proto-oncogene is characteristic of prostate cancer, and is often associated with upregulation of ribosome biogenesis and signalling. Thus, inhibition of RNA polymerase I, which slows ribosome synthesis, and of PIM kinases, which activate MYC signalling, could provide a therapeutic target.

"Prostate epithelium is exquisitely sensitive to the overexpression of the proto-oncogene MYC, which causes neoplastic transformation," corresponding author Luc Furic told Nature Reviews Urology. “Unfortunately, therapeutic agents directly targeting MYC have been largely unsuccessful, emphasizing the need to indirectly target MYC activity through inhibition of downstream cellular processes it regulates."

Furic and his team investigated the efficacy of single and dual inhibition of the ribosome, using a novel Pol I inhibitor CX-5461 and PIM kinase inhibitor CX-6258. They began with studies in immortalized prostate cancer cell lines, which express high levels of MYC. CX-5461 impaired colony formation of DU145 and PC 3 cells in culture, demonstrating that the Pol I inhibitor could suppress anchorage-dependent growth of these cells. Furthermore, they observed that CX-5461 induced cell cycle arrest in DU145, PC3, and, at a far lower dose, LNCaP cells. Treatment with the PIM kinase inhibitor CX-6258 revealed similar effects; interestingly, combination therapy with both agents significantly suppressed anchorage-dependent growth compared with the single agents.

In order to determine whether this effect would also be seen in vivo, the team went on to test CX-5461 in the Hi-MYC mouse, in which MYC expression is prostate-specific and androgen-dependent and which recapitulates the progression of prostate cancer in humans. Hi-MYC mice treated with CX-5461 displayed a significant reduction in levels of $45 \mathrm{~S}$ pre-rRNA (a marker of ribosomal transcription rate). Furthermore, repeated dosing of $\mathrm{Hi}-\mathrm{MYC}$ mice with $\mathrm{CX}-5461$ reduced $\mathrm{Ki}-67$ expression by $50 \%$
$(P=0.024)$, demonstrating reduced proliferative potential of the tumour cells and supporting the results from the cell lines.

Patient-derived xenografts (PDX) from multidrug-resistant prostate cancer metastases were used to further investigate dual therapy with CX-5461 and CX-6258, and showed a similar response to dual inhibition with significant reductions in tumour weight in the treated group of PDX mice compared with controls.

"It is noteworthy that CX-5461-CX-6258 combination is inhibitory to the growth of both androgen-receptor (AR)-positive androgen-sensitive and androgen-insensitive cell lines," comments Furic. "All these data support the conclusion of our study that AR presence or activity has no significant impact on the therapeutic activity of our novel combination therapy. Therefore, we believe our new exciting combination therapy could be used in the clinic in combination with current antiandrogens or as salvage therapy in multidrug-resistant castration-resistant prostate cancer."

Annette Fenner

ORIGINAL ARTICLE Rebello, R. J. et al. The dual inhibition of RNA Pol I transcription and PIM kinase as a new therapeutic approach to treat advanced prostate cancer. Clin. Cancer Res. http://dx.doi.org/10.1158/1078-0432.CCR-16-0124 (2016) 